\title{
Mach's Principle as action-at-a-distance in GR: the causality question ${ }^{\text {th }}$
}

\author{
Carl Hoefer ${ }^{1}$ \\ Rotman Institute of Philosophy \\ Western University \\ London, Canada \\ +1 (519) 661-2111 ext. 81291 \\ carl.hoefer@uwo.ca
}

\begin{abstract}
A part of the revival of interest in Mach's Principle since the early 1960s has involved work by physicists aimed at calculating various sorts of frame-dragging effects by matter shells surrounding an interior region, and arguing that under certain conditions or in certain limits (ideally, ones that can be viewed as plausibly similar to conditions in our cosmos) the frame dragging becomes "complete" (E.g. Lynden-Bell, Katz \& Bičák, [1]) . Such results can bolster the argument for the satisfaction of Mach's Principle by certain classes of models of GR. Interestingly, the frame-dragging "effect" of (say) a rotational movement of cosmic matter around a central point is argued by these physicists to be instantaneous - not an effect propagating at the speed of light. Not all physicists regard this as unproblematic. But rather than exploring whether there is something unphysical about such instantaneous "action at a distance", or a violation of the precepts of Special Relativity, I am interested in exploring whether these physicists' calculations should be thought of as showing local inertia (resistance to acceleration) to be an effect, with distant matter distributions being the cause. I will try to apply some leading philosophical accounts of causation to the physical models of frame dragging, to see whether they imply that the frame dragging is superluminal causation. I will then offer reflections on the difficulties of applying causal talk in physical theories.
\end{abstract}

Keywords: Mach's Principle, General Relativity, causation,

action-at-a-distance

\footnotetext{
(Title note needed?)

${ }^{1}$ Research for this paper has been supported by Spanish Ministry grants FFI2011-29834C03-03 and CSD2009-00056, SGR 2009-1528 of the Generalitat de Catalunya, and by Western University (Ontario).
}

Preprint submitted to Studies in History and Philosophy of Modern PhysicsSeptember 4, 2014 


\section{Mach's ideas on inertia}

Introduction. As is well known, a straightforward attempt to defend Leibnizian relationalism about space in a classical-physics context leads one naturally to Mach's response to Newton's bucket and globes thought experiments: the lo5 cal standard of inertial (non-accelerated) motion should be either defined, or dynamically determined in terms of the relative distances and relative motions with respect to the rest of the material bodies in the universe ${ }^{2}$ Einstein was captivated by Mach's critique of absolute space and his tentative suggestions about how to understand the phenomena of inertia without it, and capturing those ideas was an important goal as he worked toward his General Theory of Relativity (see 3]). The final theory Einstein arrived at, however, captures the Machian idea about inertia at best imperfectly. There are numerous solutions of GR in which the local metrical/inertial structure seems as absolute, or nearly as absolute, as it is in Newton's theory; and in any event, certainly not fully determined by reference to the locations and relative motions of the rest of the universe's material bodies. On the other hand, there are models of GR in which the local metrical/inertial structure everywhere does seem to be fully determined by the overall matter distribution - principally the Friedmann-Robertson-WalkerLemâitre (FRWL) "big bang" cosmological models, which are the models of GR most often taken to be good coarse-grained models of our actual universe. In addition, since Thirring's 1918 calculation of a "frame-dragging" effect in GR ${ }^{3}$ there have been numerous demonstrations of "Machian" effects in GR, that is,

\footnotetext{
${ }^{2}$ For an introductory discussion of the classical absolute $v s$ relational debate concerning space and motion, see 2], especially section 7 .

3 "Frame-dragging" is a phenomenon in which the presence of a large body nearby to a given place and moving in certain ways (especially rotating) brings about an extra 'warping' of the spacetime structure that entails that a test body moving inertially in that place will move as if there were a force exerted on it by the moving body, a force that is absent in Newtonian gravity. For example in Newton's theory a satellite in polar orbit can have the plane of its orbit stay always the same relative to the background of the fixed stars, regardless of how fast the Earth is rotating beneath; but in GR, the orbital plane will tend to rotate in the same sense as the Earth's rotation. (Because of structural similarity to magnetic induction this phenomenon in GR is sometimes called "gravitomagnetism".) Below it will become clear why this phenomenon is related to Mach's ideas on inertia.
} 
effects on the local inertial structure attributable to the relative distances and motions of large masses - a nearby planet or star, a spherical shell of matter rotating around a central point, etc. These facts have kept interest in the Machian perspective on inertia very much alive in a small segment of the GR physics community.

Among the frame-dragging results we will consider are certain recent ones published by a handful of physicists from 1995 onward, principally Lynden-Bell,

30 Bičák and Katz (hereafter LKB), using perturbative techniques in FRWL models to study what happens in GR when a spherical shell of matter is given a (small) rotation around a central point ( [1]). The intriguing results are that the framedragging effects inside the shell are instantaneous - enforced by the constraint equations on a 3-d hypersurface that follow from Einstein's equations - and, when the shell's radius becomes large enough, are complete - that is, the local inertial frames inside the shell are locked to the shell, and no longer influenced by the pre-perturbation inertial structure fixed to the matter (if any) outside the shell.

What shall we make of these results, and of Mach's idea about the origin of inert more generally? Do they represent some sort of "instantaneous action-ata-distance" - and if so, is that a problematic form of superluminal influence, in conflict with the precepts of relativity theory? Should the influence of distant matter on local inertia be considered a matter of cause and effect at all, or is some other perspective more appropriate? To set the stage for this discussion, 45 let's first address these questions in the context of Mach's famous critique of Newton with its suggestive - and not entirely clear - proposal that distant stars could take the place of Absolute Space in a proper mechanics.

Although the actual target of Newton's bucket experiment was Descartes' relational account of motion, it is possible to instead read it as an attack on 50 Leibnizian relationism and an argument for the necessity of introducing Absolute Space in order to account for inertia or (equivalently) for the so-called inertial effects present in non-inertial (accelerating and/or rotating) frames of reference, e.g. centrifugal and coriolis forces. Such a reading is especially tempting if one 
interprets the point of Newton's globes thought experiment as being to assert that in an otherwise-empty universe, we could have two globes connected by a cord which are (absolutely) rotating around their centre of mass point - with the rotation being revealed by the tension in the cord 4 In response to this way of reading Newton's Scholium thought experiments Mach wrote:

Newton's experiment with the rotating vessel of water simply informs us that the relative rotation of the water with respect to the sides of the vessel produces no noticeable centrifugal forces, but that such forces are produced by its relative rotation with respect to the mass of the earth and the other celestial bodies. No one is competent to say how the experiment would turn out if the sides of the vessel [were] increased until they were ultimately several leagues thick. (匹, p. 232.)

and earlier,

[After extensive quotes from the Scholium to Def. 8]: It is scarcely necessary to remark that in the reflections here presented Newton has again acted contrary to his expressed intention only to investigate actual facts. No one is competent to predicate things about absolute space and absolute motion; they are pure things of thought,

\footnotetext{
4 "For instance, if two globes, kept at a given distance one from the other by means of a cord that connects them, were revolved about their common centre of gravity, we might, from the tension of the cord, discover the endeavour of the globes to recede from the axis of their motion, and from thence we might compute the quantity of their circular motions. And then if any equal forces should be impressed at once on the alternate faces of the globes to augment or diminish their circular motions, from the increase or decrease of the tension of the cord, we might infer the increment or decrement of their motions; and thence would be found on what faces those forces ought to be impressed, that the motions of the globes might be most augmented; that is, we might discover their hindermost faces, or those which, in the circular motion, do follow. But the faces which follow being known and consequently the opposite ones that precede, we should likewise know the determination of their motions. And thus we might find both the quantity and the determination of this circular motion, even in an immense vacuum, where there was nothing external or sensible with which the

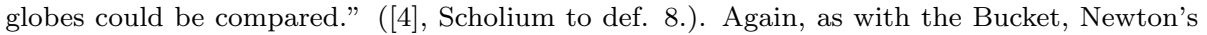
real argumentative intention here is not to assert the possibility of absolute rotation without relative motion in an otherwise-empty universe, but rather to illustrate how the quantity of absolute motions may sometimes be discoverable. But it does not seem unlikely that he would have agreed with such an assertion anyway, and readily.
} 
pure mental constructs, that cannot be produced in experience. All our principles of mechanics are, as we have shown in detail, experimental knowledge concerning the relative positions and motions of bodies. ... No one is warranted in extending these principles beyond the boundaries of experience. In fact, such an extension is meaningless, as no one possesses the requisite knowledge to make use of it. (p. 229)

And later, after having presented a suggested formula that could replace the reference to Absolute Space in the law of inertia,

$$
\frac{d^{2} \frac{\sum m r}{\sum m}}{d t^{2}}=0
$$

Mach continues his empiricist critique:

It is impossible to say whether the new expression would still represent the true condition of things if the stars were to perform rapid movements among one another.

This disclaimer seems to indicate that Mach was in no way intending to propose a genuine, new dynamical law with his inertial-frame determining equation. Nevertheless, in a late edition of the Science of Mechanics Mach displays his more speculative side:

...the natural investigator must feel the need of further insight of knowledge of the immediate connections, say, of the masses of the universe. There will hover before him as an ideal an insight into the principles of the whole matter, from which accelerated and inertial motions result in the same way. The progress from Kepler s discovery to Newton's law of gravitation, and the impetus given by this to the finding of a physical understanding of the attraction in the manner in which electrical actions at a distance have been treated, may here serve as a model. We must even give rein to the thought that the 
masses which we see, and by which we by chance orientate ourselves, are perhaps not those which are really decisive.

In 2] we distinguish two different ways of reading Mach's remarks on inertia, which we dubbed 'Mach-lite' and 'Mach-heavy'; both can be easily discerned in the above quotes. Mach-lite offers an epistemological critique of Newton's advocacy of Absolute Space, pointing out that all that experience can really teach us in our world is that there is a frame of reference - which happens to be, in our world, the frame in which the distant "fixed" stars appear to be at rest - in which Newton's three laws of mechanics hold 5 Mach-lite points out that we simply don't know what would happen if the walls of Newton's bucket were several leagues thick, or if we could somehow grab the visible stars and set them rotating around the solar system at great angular velocity. Inference to the existence of Absolute Space - or even the more modern and cautious Galilean/Neo-Newtonian spacetime - is not warranted by anything experimentally known as of the 19th century. Mach-lite therefore recommends a more cautious and relationist-sounding language when talking about inertia and motion; but strictly speaking he does not and should not claim to know that in the true mechanics of our world, relative distances, velocities and accelerations are the only space/time quantities required.

Mach-heavy, by contrast, plumps for a robust red-blooded Leibnizian relationism, and points the way to overcoming the Bucket and Globes-type considerations: Newton's dynamics should be replaced by one formulated exclusively using relationist-acceptable relative quantities (relative distances, velocities, and accelerations - implicitly, instantaneous quantities). Mach's own proposed law of inertia was not a serious attempt at offering a replacement dynamics. But attempts to modify mechanics in a way satisfying Mach-heavy in a classical framework were unsuccessful, despite the efforts of Friedländer 1896, Föpl 1904, and Reissner 1914, 1915), until the work of Barbour and Bertotti in the 1970s

\footnotetext{
${ }^{5}$ This frame, and of course all frames related to it by a transformation from the Galilean group, i.e., linear translations and rotations, and constant velocity boosts.
} 
and 80s (for details of this history see the historical articles in [6], and [7]).

Aversion to Mach-heavy. The Mach-heavy side of The Science of Mechanics clearly inspired not only the above mentioned physicists, but also and more famously, Albert Einstein. On the other hand, negative reactions to Mach-heavy have not been scarce, either among physicists or philosophers (for a prominent example, see [8]). One frequently raised complaint is especially relevant for the rest of this paper: the dynamical connections between massive bodies bruited in Mach-heavy would seem to amount to a new, complicated, and extremely hard-to-test instantaneous action-at-a-distance. The connection must, apparently, be instantaneous because, from the naïve relationist perspective, I can instantaneously set all the universe's masses into rotation about me (by spinning in ice-skater fashion). The centrifugal and coriolis forces arise instantly, not after a delay during which a supposed influence of the change in relative motions might travel out from my body, be registered by the distant stars, and cause a corresponding response which in turn propagates back to my (by now long-decayed) body.

There is an alternative interaction picture that could explain the instantaneous appearance of centrifugal and coriolis forces. One could imagine the masses of the universe to be constantly "emitting" some causal influence that travels outward at the speed of light, all these influences joining together to generate the inertial structure felt at any particular place. Notice that in this picture it is not the instantaneous relative rotation of my body with respect to the other masses in the universe that is responsible for the appearance of inertial forces; it might be said however that it is my apparent rotation - my rotation relative to the incoming light from the stars - that correlates with the inertial forces. We will touch on this alternative picture again later.

Mach's discussion of relative motion is, unsurprisingly, framed in terms that presuppose the absoluteness of coexistence, i.e., absolute simultaneity: for a given body $\mathrm{A}$ at a moment of time, there simply are facts about the relative distances between A and all other existing bodies in the universe, and about 
their relative velocities and accelerations - which facts can be expressed in a coordinate system rigidly fixed to A, or to any other body ${ }^{6}$ In the post-Special Relativity context, of course, the absolute simultaneity structure assumed by Mach in his discussion is rejected, and this apparently problematises the whole idea of the Mach-heavy explanation of inertial forces 7 Doesn't Special Relativity forbid causal influences that propagate faster than the speed of light? And if it is a genuine influence could it not be used to send instantaneous signals, raising the spectre of faster-than-light communication and the attendant causal paradoxes?

One reaction to this worry might be to say: "Not so fast!". First, one may question whether functional dependence or determination between co-existing things (whether that be gravitational force dictated by Newton's law of gravity, or inertial forces determined a là Mach by relative distances and motions) should automatically be considered a matter of causation. Secondly, one may note that even assuming that spacetime has a Lorentzian structure, that does not automatically guarantee the physical possibility of producing the kind of zig-zagging space-like causal connections needed to produce causal loops (see 9] sec. 3.3). And finally, it is worth noting that what Special Relativity profers, General Relativity may take away. GR does not have the same symmetry group as SR. The symmetry group of all special-relativistic theories is the Poincaré group, whereas for GR, under one perspective the symmetry group is the general group of continuously differentiable diffeomorphisms, and under a different perspective the theory has no spacetime symmetries. Many models of GR have dynamically distinguishable preferred frames in which the spacetime structure

\footnotetext{
${ }^{6}$ The spacetime structure implicitly assumed by Mach and earlier thinkers is nowadays usually called "Leibnizian spacetime" (see [8]). In Leibnizian spacetime there is absolute simultaneity and an absolute temporal metric, but no preferred states of motion, e.g., no distinction between accelerated/non-accelerated or rotating/non-rotating frames.

${ }^{7}$ In the post-General Relativity context Einstein and others have asserted that another big change in the world's presumed ontology also problematises Mach-heavy: the rise of the field as the primary physical entity in spacetime, replacing the "ponderable masses" that are assumed to be the dominant material constituents of the universe in both Mach's and Einstein's early discussions of inertia. I will not address this second difficulty for the Mach-heavy idea about inertia in this paper.
} 
has a particularly simple expression, in which (among other things) clocks run at a faster rate than in all non-co-moving frames. And some models of GR contain closed time-like paths, so the theory has potential troubles from causal paradoxes independently of any Machian instantaneous action-at-a-distance.

But another way to address the raised concern is to explore more carefully the question: Is the Mach-heavy approach to inertia necessarily a causal explanation in the first place? In the rest of this paper I will address this question, both as a question for the Machian idea in general, and in particular in light of the complete-frame-dragging results mentioned earlier.

\section{2. "Mach's Principle" cashed out?}

The FRWL Big Bang models of GR are prima facie hospitable to a Machian account of inertia. There is a homogeneous distribution of matter throughout the universe, with the local inertial frames anchored as expected to the cosmic matter's rest frame; there is no global rotation of the matter, nor any empty region "at infinity" to partly determine or influence the inertial structure in central regions. Although these facts hold as much for the infinite $(k=0$ and $k=-1)$ as closed finite $(k=+1)$ universes, relativists favourable to a Machian account of inertia have traditionally singled out the $k=+1$ universe as especially clearly Machian in character (see [10, [1). In more recent works using rotational perturbations of FRWL models, however, Machian "complete" frame-dragging results have allegedly been proven for $k=0$ and $k=-1$ cosmologies as well as $k=+1$ ([1], [1]). Before we turn to these rotational-perturbation studies, however, it will be instructive to consider a super-simplistic argument for the Machian character of FRWL universes - one that harks back to Einstein's own initial thoughts about the generalised relativity of motion.

The simplistic argument starts by reconsidering Mach's polemic against Newton. Mach asks us to imagine putting the whole sphere of the fixed stars into rotation around us, and then prove that there will be no centrifugal forces 
could be easier than putting the whole universe into rotation? We just need to transform our description of the actual cosmological situation into coordinates rotating around the chosen central point at the appropriate angular velocity. But Mach's challenge involves a situation in which the water is non-rotating in is a mechanics that treats all reference frames equally $a b$ initio and does not introduce materially-inexplicable corrective forces when we use certain frames to describe a situation. In other words, a mechanics whose equations are valid and can be applied, without corrections, in any coordinate frame of reference we (i) generally covariant and (ii) having no background spatiotemporal structures, 
is just such a theory! And in fact this was how Einstein saw things at times in the years 1913-16: Implementing (i) and (ii) ought to be sufficient to guarantee the general relativity of motion in just the sense desired by Machian relationism.

Whether, how and why this line of thought goes astray in general is a subject that has been discussed thoroughly in the literature and I will not pursue the general issue here (see e.g. [2]). But let's apply the thought to FRWL cosmologies as a particular case. In order to prove that Mach's ideas are fully satisfied in these cosmologies, perhaps it is enough to simply transform the (coordinate-based) solutions into coordinates rotating around a central point and note that precisely the effects Mach posited should occur, do occur! The centrifugal forces in the rotating frame are simply the result of the way that geodesics curve and twist in such a frame, of course: the metric, and hence the inertial structure, when described in such a frame have various important terms that are not present in the expression of the metric in the co-moving frame. These are counterparts of the Newtonian's pseudo-forces. But rather than having their origin in something absolute and theoretically unrelated to the positions and movements of matter in the world, now they have their origin in the spacetime metric which - plausibly at least - is determined by the distribution of matter-energy around the rest of the world, and its motions (as described in the chosen coordinate frame). In other words, it can be argued at least that all FRWL cosmologies satisfy Mach's ideas about the origin of inertia perfectly well, and that this can be seen by simply transforming the solution of Einstein's field equations into a rotating coordinate system.

This simplistic argument does not work for models of GR in general. Take for example a model that describes something like our Earth, in an otherwise empty universe. The GR description of such a system - essentially the Schwarzschild exterior metric, or the Kerr metric - involves a spatially infinite spacetime which approaches flat Minkowski metrical structure far from the central bodies. Now if we describe things using a coordinate system anchored (in some sense) to a whirling ice-skater on the Earth, once again by transforming the metric around the skater into these new coordinates we will find centrifugal forces popping into 
existence just as they should. But now these forces are not plausibly attributable to the existence of huge amounts of cosmic matter rotating quickly around the skater, for there is no such matter out there. There is only the planet under her feet; and the size of the centrifugal forces does not vary much as the mass of the Earth is set higher or lower; it is instead almost entirely a function of the rate of rotation of the skater relative to frames in which the Minkowski metric far away takes its usual simplest form. In other words, it is the (quasi-) absolute spacetime far away from the central bodies that is (it would seem) almost entirely responsible for the centrifugal forces appearing in a frame fixed to the skater, and not any relative motions of ponderable material bodies in the world.

The difference is clear: for FRWL models no boundary conditions at infinity are imposed - either because there is no infinity $(k=+1)$, or because there $i s$ a metrical structure infinitely far away from our chosen central point, but the metric there is identical to the metric at the centre, and hence just as relationally determined "out there" as it is anywhere else 8

The simplistic argument for the fulfilment of Mach's ideas in FRWL models depends on a controversial assumption, as we have seen: that one be able to consider the metric and inertial structure as (in some sense) everywhere determined by the matter distribution (local and distant).

Sidebar: metric and matter-energy. A few notes about the idea of the metric being 'determined by' the distribution and motions of matter-energy are in order

\footnotetext{
${ }^{8}$ This reflection helps see why one complaint sometimes raised against the Machian idea concerning inertia in the context of GR is misguided. If we describe the world using a coordinate frame fixed to the skater, then most of the world's matter (perhaps the Moon, but certainly Mars and everything else further away) will be moving at speeds greater than $c$, the speed of light, in apparent defiance of Special Relativity. Surely the whole idea is absurdly un-physical?

But in fact this is a pseudo-problem. The far away bodies rotating around the skater do not move superluminally, because in the chosen coordinate frame, the light-cone structure itself is tilted strongly over in the direction of the rotation of the matter. The far away stars and galaxies move along perfectly nice geodesics of the metric, inside the local light-cone structure. And as long as there is matter filling space, as in FRWL models, this tilting of the light cones is (arguably?) a perfectly intuitive frame-dragging "effect" of the high-velocity motion of such large amounts of matter.
} 
in GR. Mathematically speaking a superficial reading of Einstein's equations $G_{\mu \nu}=8 \pi T_{\mu \nu}$ suggests that the direction of determination is just the opposite. For given the metric everywhere, Einstein's tensor $G_{\mu \nu}$ can be calculated, and therefore trivially also the stress-energy tensor. Moreover the metric is required to give meaning to $T_{\mu \nu}$, so to speak; simply giving a set of 10 functions of on a spacetime manifold and calling it ' $T_{\mu \nu}$ ' does not amount to saying how much rest mass (density), stress, or pressure there is, at a point or region, or how it is moving. One has to know the metric field in order to interpret $T_{\mu \nu}$ physically (see Lehmkuhl [12 for a thorough discussion). not meant to be a fundamental description of matter; it is instead a field that represents, one might say, the 'gravitationally relevant' aspects of matter fields that are described more literally off-stage in some other theory (e.g. some relativistic field theory). While it is true that specification of the metric field notions of local energy density, pressure, temperature etc. are independently meaningful, and to some extent operationally determinable by physical measurements. This is in fact related to the Equivalence Principle: local experiments and measurements in a closed lab won't reveal whether one is in otherwise-empty Minkowski spacetime, or traveling inertially in a FRWL cosmos, or falling (at a safe distance!) toward a Schwarzschild black hole; but one can perfectly well measure how much 'ordinary' matter and energy of various kinds are present in the lab ('ordinary' being a qualification that excludes, at least, gravitational field stress-energy). And coming back to the superficial reading of Einstein's not a "supersubstantivalist" theory that reduces matter to being a structural property of spacetime. One should not think of the metric field as fully determining (in a conceptual or physical sense) what the matter-energy content distribution in a world is. What is more plausible is that Einstein's equations capture relations of mutual influence and dependence between matter-energy 
present in the world and the structure of spacetime. The Machian perspective simply requires that - in some kinds of worlds at least - the dependence be plausibly seen as predominantly from matter-energy distribution to spacetime (especially inertial-motion) structure.

'Complete' determination of inertial structure by matter-energy?. That the dependence of spacetime structure on how mass-energy is distributed around can in some cases look plausibly complete was mentioned above already for the case of FRWL cosmologies, for which no metrical boundary conditions have to be imposed to derive the solution of the full metric; the spatial homogeneity and isotropy of the (simple, idealised) matter-energy content, plus quantities such as mass density and pressure, is enough, given Einstein's equations, to determine the metric everywhere. So let's grant for sake of argument that the idea of the metric and hence inertial structure being 'determined' by the distribution of matter-energy can make sense, for at least some models of GR. Then this perspective is surely only strengthened if one can show that a bunch of cosmic matter moving in a frame of reference (accelerating, rotating, etc.) will somehow 'drag along' the metrical structure with it - and, indeed, completely drag it along, if there is enough of it. The increasing-frame-dragging results pursued by Mach-sympathetic physicists can be seen as providing this reinforcement. First, they show the desired creation of centrifugal etc. forces in the centre by spheres or rings of matter rotating around; and further, they try to show that the idea of complete dragging is physically real, not merely achievable by coordinate change tricks; and because when complete dragging is achieved at the centre the "distant masses" at infinity no longer have any influence - hence, neither would 350 a quasi-Minkowski background or boundary-condition - they make it plausible that in such a model the metric is indeed, in a relevant sense, everywhere "determined" just by the matter distribution and its relative motions.

It is the "complete" dragging that may be questioned, because that there is at least some frame-dragging caused by all nearby rotating/accelerating material bodies is something that was demonstrated for GR by Einstein in rough 
calculations quite early on, and more precisely in works by Thirring in 1918 ([13]), which demonstrated induced forces both inside and outside a rotating mass shell. After this early work relatively little new was proven until the 1960s, when Brill \& Cohen ([14]) studied how a little bit of frame dragging e.g., by a shell of matter rotating around a central point, set in a spacetime with well-defined boundary regions that are non-rotating - becomes bigger and bigger as the size and mass of the shell increases, ideally yielding "complete" dragging of the inertial compass in the centre of the shell after some threshold has been crossed. (Notice that these calculations are in essence the working out in GR of Mach's hypothetical situation in which the walls of Newton's bucket are "several leagues" (or megaparsecs!) thick.)

Brill \& Cohen showed that a rotating rigid mass shell induces frame dragging in the centre, which dragging becomes complete (interior inertial frames locked to the shell) once the mass of the sphere is large enough and close enough to all be within its own Schwarzschild radius 9 But the shell of matter built into their model was not physically realistic, involving infinite stresses in the shell at the limit where perfect dragging is achieved. Related work by Lindblom \& Brill in 1974 ([16]) used a more realistic thick shell consisting of rotating and at the same time in-falling matter and showed that the frame dragging at the 375 center of the shell is most naturally interpreted as being "instantaneous" - that is, determined at the centre by the state of rotation of the shell at the "same time" in the chosen coordinate system, rather than being related to the retarded rotation rate. Interestingly, Lindblom and Brill themselves seem to speak of this as a matter of more/less natural interpretation, and devote a couple of sentences to disarming possible concerns about violation of the prohibition of superluminal causality:

That is, as seen from infinity, the inertial frames within the shell rigidly rotate at the angular velocity $\Omega_{o b s}$ : There are no retardation

\footnotetext{
${ }^{9}$ These results were extended to 2 nd order in $\omega$, the angular rate of shell rotation, by Pfister \& Braun ([15]) in 1985.
} 
effects between the shell and the inertia of a gyroscope at its center.

This of course does not contradict any physical causality principle, since $\Omega_{-}$[the 'induced' rotation of interior inertial frames] can be considered to be merely the angular velocity of a coordinate system for the interior flat region. However, it is this coordinate system which is most directly related to effects observable from infinity, as explained above. Thus another view, more closely related to Machian ideas, is equally consistent, in which $\Omega_{-}(t)$ is observable but highly nonlocal, so that a local causality principle does not apply to it. $([16$, p. 3154$)$

It is not made clear why an observable phenomenon being considered "highly nonlocal" means that local-causality principles need not apply!

The main newer model calculations of interest to us depart from a 1995 paper by Lynden-Bell, Katz \& Bičák (LKB) in Mon. Not. Roy. Astr. Soc. (1]), and include subsequent papers by these authors and by C. Schmid, mostly published in Phys. Rev. D. These later works provide models of "perturbed" FRWL cosmologies in which a shell of matter is given a (small) angular velocity, and the consequent frame dragging in the centre is calculated. LKB initially claimed full satisfaction of Mach's Principle only for $k=+1$ universes, but in later papers - written partly in response to work by Schmid (esp. [1]) - they agree with Schmid that relevant complete-dragging results can be obtained for any FRWL universe:

For given distributions of energy-momentum and angular momentum of matter sources, the rotations and accelerations of local inertial frames are uniquely given in the Machain gauges in open universes under suitable boundary conditions, whereas in closed universes they are determined up to motions generated by the Killing vectors, i.e., by symmetries of the background. They are determined uniquely also in closed universes if velocities, density, and pressure perturbations of cosmic fluid are given. As a consequence of the con- 
straint equations and the choice of gauges which imply suitable slicing of perturbed universes, these inertial properties are determined instantaneously. In this sense Machs principle is embodied in the cosmological linear perturbation theory. ([17], in ArXiv:0803.4106v1, p. 55)

The complexity of the calculations in these papers is in general quite high, unsurprisingly given the nonlinearity of GR and the difficulties of working with three distinct background metrics (and two distinct topologies). The interpretation of various equations and results can also be controversial, it being especially difficult to single out a mathematical representative for the relative rotation between a local inertial frame in the center and the perturbed/rotating shells. Such a quantity is inevitably in part coordinate- or frame-dependent, and its aptness for representing the extent to which the local inertial compass is "dragged along" by the rotating cosmic matter something open to discussion and debate.

The perturbative calculations fall short of offering a definitive exploration of the nature and extent of interior frame dragging by matter shells that one might ideally wish for, to bolster the case for satisfaction of Mach's ideas on inertia by FRWL (and other) models of GR. The solutions arrived at are valid only to 1st order in general, and do not offer a complete 4-d model of a situation that satisfies the Einstein field equations over time. Being tied to particular choices of 'gauge' or coordinate system, they also require careful treatment to sort out measurable and physical phenomena from mere coordinate-choice artefacts. Furthermore, although some application of these techniques to 'retarded' rotations - rotation of a matter shell found on the backward light-cone from the chosen central point - has begun ([18? ], [19]), there has been as far as I know no real exploration of the status of retarded vs "instantaneous" frame dragging results, or what to say if (as seems not implausible) complete frame dragging results can be found for rotating matter on well-chosen simultaneity slices, on the backward light cone, and even on the forward light cone. As LKB 
aptly say in (2008, p. 56), "A thorough nonlinear study of Machs ideas within

\section{Is Machian frame-dragging causation?}

A simplistic way of approaching this question, regarding the "instantaneous" dragging results at least, might go like this: "These calculations link the state of affairs at far-away regions on a spacelike hypersurface with the state of affairs equally importantly, the frame-dragging relations under discussion here are precisely a potential counterexample to the premise. Insofar as we understand 'causation' in a way that does not analytically rule out superluminal causation, the simplistic response is completely unsatisfactory.

And although the concept of 'causation' discussed by philosophers (and widely used in physics, as well as daily life) is notoriously hard to define or analyse, and fraught with controversy, it is clear enough that no superluminal causation is at most a true fact about our world, not a part of the meaning of 'causation' 10 How, then can we approach the question of whether Machian

\footnotetext{
${ }^{10}$ In a sense this is obvious just from the fact that many philosophers and physicists considered gravity to be an instantaneous causal action at a distance while also supposing that light might travel at a finite speed. But even setting aside classical spacetime-based intuitions and taking the semi-Riemannian structure of spacetime as conceptually fixed, that no
} 
causal influence can travel outside the light-cone structure is just not a conceptual truth; the contemplatibility of both tachyons and time travel shows this.

to approach this question from the perspective of some of the most influential philosophical accounts of causation. Without letting this approach turn into a plodding exercise, I want to consider how things look from the perspective of a Lewis-style counterfactual theory of causation and from that of Jim Woodward's manipulationist theory $(20])$.

But before doing so, we can pause to note that two other influential contemporary approaches to causation are of no use here. (1) The Salmon-Dowe process theory of causation is often considered possibly a good account of causation for basic physical relations, even though it appears inapplicable to many important domains where causal talk is ubiquitous, such as history and economics. But as I have noted elsewhere (21], ch. 34), the most recent, "conserved quantity" version of that theory is not clearly well suited to the context of GR, where quantities such as energy (or mass-energy) momentum and angular momentum are in general neither well-defined nor conserved. It seems clear that there is no continuous causal process possessing a conserved quantity linking the rotating matter shell to the interior inertial structure. But there is also no causal process possessing a conserved quantity linking a supernova to a gravity-wave detector constructed to detect the gravitational waves produced by such explosions, or even one linking a binary-star system at one moment of time to the same system a year later - in both cases, because there is nothing strictly conserved in the linking processes. (2) In recent years mechanistic approaches to causation have been championed by Glennan, Machamer, Craver and others, and have been widely discussed (see ([21], ch. 15). But even the advocates of mechanism admit that it is not applicable to the level of fundamental physics (this is sometimes called the "bottoming out problem"). And it is surely clear that even if we decide that Machian instantaneous frame dragging is a matter of cause-effect, it will not be because we discern a mechanism that carries the 
influence/connection 11

Let's consider Machian frame-dragging from the perspective of counterfactual and manipulationist theories of causation. We can start with the oldest and simplest of such effects, the "Lense-Thirring" effects mentioned earlier 12 In these scenarios a stationary rotation is set up, and a dragging force is calculated on freely moving test bodies inside or outside the rotating spherical mass. Since in the case of zero rotation (relative to the Minkowski boundary conditions) there is (clearly, by symmetry considerations alone) no dragging force present, the counterfactual test seems to be clearly passed: the rotation of the big spherical mass causes the dragging effects, interior and exterior. Things are also straightforward on the manipulationist approach. If one were to intervene on the putative cause (rotating mass), say by increasing or decreasing its rate of rotation a bit, the Thirring calculations show precisely how much the dragging effects would be correspondingly increased or decreased. And such an intervention seems straightforwardly to meet the criteria of Woodward's theory, hard though it may be to put into practice on a planetary or stellar scale. (One might for example use carefully arranged H-bomb detonations to fractionally speed up or slow down the Earth's rate of rotation relative to the fixed stars.) Things are however more complicated when we consider cosmological models, and thinking about the complications will lead us to see that the causality question may not be as open-and-shut as it seems even in the case of small local frame-dragging effects.

Central to applying either a Lewisian counterfactual theory or the Woodward theory is setting up a counterfactual situation that differs with respect to the cause-variable and then using physical theory (and/or other theories, com-

\footnotetext{
${ }^{11} \mathrm{By}$ this I mean that there is clearly no mechanism linking the cause to the effect, in the description provided by GR. It remains an open possibility that a future theory could supersede GR and offer an account of gravity and inertia that involves heretofore-hidden mechanisms. If something like that were developed, it obviously might answer the question of whether Machian frame-dragging is a transmitted (and hence 'retarded') effect, or not.

${ }^{12}$ Pfister (7] argues that Lense's role in the early discovery and calculation of these effects was rather small, hence the scare quotes around "Lense-Thirring effect" here (which will be dropped for the rest of the paper.)
} 
mon sense, etc.) to answer the question of whether/how things would then be different for the effect-variable. In a question such as ours, involving a physical theory like GR, there are different ways one might try to go about modelling and computing things to answer the question. Typically what is manageable is to find two distinct solutions that satisfy the theory's laws or equations, and treat them as though the 2 nd one represents the appropriate counterfactual scenario or result of intervention. But in fact the models one can most easily compare (FRWL model vs perturbed model; static sphere vs stationary-rotating-sphere model) do not necessarily answer to the desiderata of the philosophical accounts of causality we are considering.

For both a Lewisian and a Woodwardian approach, the counterfactual change or intervention should be restricted in scope: grab (e.g.) some matter and set 535 it rotating (or change its rate of rotation), but leave everything else untouched, then use the theory's equations to evolve forward a consistent solution to see what 'effects' ensue. Ideally this would mean using the initial value formulation (IVF) of the theory: set up things on a 3-slice in the way demanded by the relevant counterfactuals and let Einstein's equations in the IVF tell us how things evolve from there. But here we have to take note of a problem: the constraint equations that make up part of Einstein's equations - precisely the equations used in the "instantaneous" frame dragging calculations described earlier - prevent us from doing the kind of surgical counterfactual changes that are ideally desired for applying our theories of causation.

Philosophers may recall Lewis' metaphysical principle of composition that is a key part of his Humean Supervenience: using the approved 'perfectly natural kinds' defined at points or small regions of spacetime, one may arbitrarily construct situations - e.g., lay out a state of affairs on a spacelike surface - by distributing those natural-kind properties however one likes over space. This 550 is what happens in the notorious "small miracles" invoked to change an actual situation to a counterfactual one, ignoring the fact that if the world's laws are deterministic (enough) the new situation is likely to be dynamically inconsistent with the actual past. Ignoring that inconsistency, one forges ahead - in time - 
to see whether the effect still occurs. Clearly, one can't forge ahead using GR's again, one cannot use GR sensibly to compute the results of the intervention without making the whole situation on the initial hypersurface consistent with Einstein's equations, one has to modify the metric everywhere on the hypersur- 
face. And doing so directly entails the putative effect, frame-dragging at the centre, this being precisely what the calculations of LKB and others demonstrate. On the one hand one could say "Perfect! Intervention on the causevariable entails change in the effect-variable, so we have here causation." On the other hand, a critic could question whether the spirit of the notion of an intervention isn't actually violated here. For if distant matter rotating (or not) is a putative cause of the inertial frame at the centre, surely the state of the spacetime metric closer-in is, too, as is the metric further away, or "at infinity" (for scenarios where such a boundary condition exists). For example, if inside or outside of the matter shell whose rotation constitutes our intervention we had strong gravitational waves circulating in the opposite sense, that would reduce or nullify the net effect - or at any rate, clearly modify it. So the presence or absence of gravity waves, or more generally speaking the state of the metric field, in other regions must be considered another putative cause of the inertial structure at the centre; and so, to have a Woodwardian intervention on the matter shell, one would have to be able to modify it while leaving unchanged the metric elsewhere. This being impossible, a critic could well argue that frame-dragging models of the kind we have been contemplating simply fail to be tractable under Woodward's theory, and hence no clear verdict on the question of causality is possible.

${ }_{605}$ Do these considerations equally undermine our initial assessment about LenseThirring-type frame dragging in the interior or exterior of a star or planet? I believe they do not, and the reason is connected to the difference between using a model of GR to describe a small, local phenomenon in an overall cosmological situation that can be ignored or left off-stage, vs. using a model of GR to directly represent the whole universe. In that initial assessment we compared a simple non-rotating-planet model to a stationary rotating-planet model; neither perturbations nor the initial value formulation came into play. The distinct metrics of the two models are simply taken as being good local approximations for the metrics near to a non-rotating/rotating planet (respectively), embedded 15 in a world that globally, far away from the planet in question, is for all prac- 
tical purposes identically the same either way. In a strict mathematical sense, the rest of the cosmos cannot have exactly the same metric elsewhere, but the differences are so minute as to be negligible. And both the counterfactual and manipulationist approaches are willing to set aside negligible differences where appropriate. So there is (arguably at least) no difficulty in getting the appropriate counterfactuals (for the Lewis approach) or in describing a proper intervention (for Woodward's) and deriving the requisite results.

In the case of Lewis one can think of the "small miracle" that changes the planet from non-rotating to rotating (or changes its $\omega$ ) as being a simple cutting and pasting together of two models of GR: the lower half coming from the first (e.g. non-rotating) model and the upper half (after time $t$ in some appropriate coordinate frame) from the second, rotating model. The miracle is in effect small, since it imposes only negligible change on the metric field far from the planet ${ }^{13}$

In the case of Woodward's approach the difficulty that an intervention may not be physically possible without changing things in such a 'big' way that other causally relevant factors are not left alone is explicitly discussed by Woodward, using (as it happens) an example involving planetary-scale gravity: the distance of the Moon from the Earth as a causal factor for the size of the tides 14 Woodward does not require that an intervention be physically possible, but rather only logically or conceptually possible. In the case of doubling the Moon's distance to the Earth, he takes Newtonian mechanics itself to supply the required counterfactuals about what would happen to the tides - by giving us the alternate stationary-state model of an Earth-Moon-like system where the Moon is twice as far away. The intervention does not need to be directly captured

\footnotetext{
${ }^{13}$ Undesirable backtracking counterfactuals (ones in which the consequence is a change in the past given some change in the present state) are avoided in the standard way by Lewis' criteria for world-similarity.

${ }^{14}$ Using a term borrowed from Sober, Woodward describes an actual or physically possible intervention like this as 'ham-fisted'. The idea mooted above of using H-bomb detonations to change the Earth's angular velocity $\omega$ might well be too ham-fisted to serve as a Woodwardian intervention (if, e.g., the stress-energy of the explosions has to be so non-negligible that it is not reasonable to ignore its direct effect on the metric nearby).
} 
as a physically possible event representable in a model of the theory. In our local frame-dragging case, we can again take the simple non-rotating model to represent the pre-intervention situation and the rotating model to represent the post-intervention situation. Since the other causal factors influencing inertial motion near the planet, especially the metrical boundary conditions far away, are only negligibly affected by the switch from the first model to the second, Woodward's notion of a proper intervention appear to be satisfied.

Matters are quite different, however, when we are talking about intervening on a substantial fraction of the material contents of the world, and producing a complete frame-dragging result. The cosmic shape of the metric outside the rotating shell (and "at infinity" where applicable, i.e., in non-closed models) cannot be ignored and treated as unaffected, because in the limit as the matter shell grows, the frame dragging must become complete everywhere, not just in the interior. The sizes of the (putative) causes and effects are decidedly non-negligible!

Finally, in the case of frame-dragging near a planet we may wonder whether, if we decide that it is clear that it amounts to causation according to counterfactual and manipulationist theories, it is also clear whether or not the cause-effect relationship is "instantaneous" or rather retarded. Unfortunately, the simplistic, compare-two-4-D-models approach to setting up the counterfactual situation/intervention does not let us answer this question. One model is taken to be a good representation of what the metric is like near a non-rotating planet (static situation), another is taken to be a good representation of what the metric is like near a rotating planet (stationary situation) well after intervention or the small miracle. If we imagine a real-world intervention that puts a planet into rotation (or changes its angular velocity $\omega$ ), these models serve well to capture the pre-intervention situation and the post-intervention situation - after things have settled down, that is. But this pair of models does not in any way capture what the metric would be like during the course of such an intervention. Physical intuition may lead us to expect that creation or change of frame-dragging effects would propagate outward from the intervention site(s) at the speed of 
light, and not be felt instantaneously; but as far as I know there are no models of GR that cover such a changing-rotation situation both realistically and rigorously.

Taking stock. What is the upshot of all of the above applications of philosophical theories of causation to Machian frame-dragging? While small local frame-dragging effects seem to clearly count as instances of causation, the picture when it comes to the allegedly instantaneous frame-dragging in cosmological models (Lindblom \& Brill, LKB, Schmid) is decidedly murky. Both the standard Lewis-style counterfactual theory and Woodward's manipulationist theory are arguably not applicable: the basic recipes for setting up the relevant counterfactual scenarios are difficult or impossible to implement.

It's not that any specific counterfactual statement is itself difficult to assess; rather, it's only difficult to tell whether one can satisfy the input-requirements for the philosophical theory of causation. The situation reminds me strongly of what Christopher Hitchcock notes concerning traditional puzzle-cases for philosophical accounts of causation ("Of Humean Bondage", [22]). The underlying facts that the are used as input by such accounts are typically perfectly clear and agreed-upon. (Which rock actually struck the window, Billy's or Suzie's? What would have resulted had the initial conditions been slightly different, in such-and-so manner?). The underlying facts are not controversial; what gets disputed is just the philosophers' question "Do we have causation here?". In that paper Hitchcock recommended that we stop trying to answer that question by producing new and improved theories of causation. While I'm not sure I want to sign up for this skeptical/deflationist attitude across the board, I suspect it is the right one to take when it comes to puzzling questions of causality in GR, such as our frame-dragging question, or the vexed question of whether the metric field causally affects bodies moving inertially.

If we adopt such a deflationist attitude concerning Machian effects in GR, then interestingly, the distinction between Mach-lite and Mach-heavy discussed in section 1 can be minimised if not eliminated entirely. What Mach wanted, expressed in terms appropriate to GR, is a perspective from which one has a 
dynamics with no absolute background (a given, in FRWL models) and one can see the local inertial structure as in some appropriate sense "determined"

for now the Mach-heavy perspective can be considered partially vindicated in GR, and more vindicated the more we deflate it into something close to Machlite. But in GR, at least, a causal understanding of Machian frame-dragging at 
the cosmological scale is certainly not forced on us.

[2] N. Huggett, C. Hoefer, Absolute and relational theories of space and motion, in: E. N. Zalta (Ed.), The Stanford Encyclopedia of Philosophy, fall

[3] C. Hoefer, Einstein's struggle for a machian gravitation theory, Studies in History and Philosophy of Science Part A 25 (3) (1994) 287 - 335. doi:http://dx.doi.org/10.1016/0039-3681(94)90056-6. URL http://www.sciencedirect.com/science/article/pii/ 0039368194900566

[4] I. Newton, Philosophiæ Naturalis Principia Mathematica, 3rd Edition, Vol. I, University of California Press, Berkeley, CA, 1726, the translation by A. Motte of the third edition (1726), originally produced in 1729 , revised by F. Cajori and published in 1934. The first edition of the Principia was published in 1686, the second in 1713 .

[5] E. Mach, T. McCormack, The Science of Mechanics, Cambridge Library Collection - Physical Sciences, Cambridge University Press, 2013.

URL http://books .google.com/books?id=IdD1AAAAQBAJ

q [6] J. Barbour, H. Pfister, Mach's Principle: From Newton's Bucket to Quantum Gravity, Einstein Studies, Birkhäuser Boston, 1995.

URL http://books .google.com/books?id=fKgQ9YpAcwMC 
[7] H. Pfister, On the history of the so-called lense-thirring effect, General Relativity and Gravitation 39 (11) (2007) 1735-1748. doi:10.1007/ s10714-007-0521-4.

URL http://dx.doi.org/10.1007/s10714-007-0521-4

n [8] J. Earman, World Enough and Space-Time: Absolute Vs. Relational Theories of Space and Time, Bradford Books, MIT Press, 1992.

URL http://books .google.com/books?id=g2zbKQAACAAJ

[9] J. Butterfield, Reconsidering relativistic causality, International Studies in the Philosophy of Science 21 (3) (2007) 295-328. arXiv:http://dx.doi. org/10.1080/02698590701589585, doi:10.1080/02698590701589585. URL http://dx.doi.org/10.1080/02698590701589585

[10] C. Misner, K. Thorne, J. Wheeler, Gravitation, Freeman Press, San Francisco, 1973.

775 [11] C. Schmid, Cosmological gravitomagnetism and mach's principle, Phys. Rev. D 74 (2006) 044031. doi:10.1103/PhysRevD.74.044031. URL http://link.aps.org/doi/10.1103/PhysRevD.74.044031

[12] D. Lehmkuhl, Mass-energy-momentum: Only there because of spacetime?, The British Journal for the Philosophy of Science 62 (3) (2011) 453-488. arXiv:http://bjps.oxfordjournals.org/content/62/3/453. full.pdf+html, doi:10.1093/bjps/axr003. URL http://bjps . oxfordjournals .org/content/62/3/453.abstract

[13] H. Thirring, Über die wirkung rotierender ferner massen in der einsteinschen gravitationstheorie, Physikalische Zeitschrift 19 (33).

785 [14] D. R. Brill, J. M. Cohen, Rotating masses and their effect on inertial frames Phys. Rev. 143 (1966) 1011-1015. doi:10.1103/PhysRev.143.1011. URL http://link .aps.org/doi/10.1103/PhysRev.143.1011 
[15] H. Pfister, K. H. Braun, Induction of correct centrifugal force in a rotating mass shell, Classical and Quantum Gravity 2 (6) (1985) 909. URL http: //stacks . iop.org/0264-9381/2/i=6/a=015

[16] L. Lindblom, D. R. Brill, Inertial effects in the gravitational collapse of a n rotating shell, Phys. Rev. D 10 (1974) 3151-3155. doi:10.1103/PhysRevD. 10.3151

URL http://link.aps.org/doi/10.1103/PhysRevD.10.3151

795 [17] J. c. v. Bičák, J. Katz, D. Lynden-Bell, Cosmological perturbation theory, instantaneous gauges, and local inertial frames, Phys. Rev. D 76 (2007) 063501. doi:10.1103/PhysRevD.76.063501. URL http://link .aps.org/doi/10.1103/PhysRevD .76.063501

[18] C. Schmid, Exact Dragging of Inertial Axes by Cosmic Energy-Currents on the Past Light-ConearXiv:1406.4665.

[19] B. Vetö, Retarded cosmological gravity and Mach's principle in flat FRW universes arXiv: 1302.4529 .

[20] J. Woodward, Causation and manipulability, in: E. N. Zalta (Ed.), The Stanford Encyclopedia of Philosophy, winter 2013 Edition, 2013.

805 [21] H. Beebee, C. Hitchcock, P. Menzies, The Oxford Handbook of Causation, Oxford Handbooks in Philosophy, OUP Oxford, 2009.

URL http://books.google.com/books?id=BXyjngEACAAJ

[22] C. Hitchcock, Of humean bondage, The British journal for the philosophy of science 54 (1) (2003) 1-25. 